\title{
Lidil
}

Revue de linguistique et de didactique des langues

33 | 2006

La réception des textes littéraires

\section{École et dégoût littéraire}

\section{Michel P. Schmitt}

\section{Q OpenEdition \\ Journals}

Édition électronique

URL : http://journals.openedition.org/lidil/70

DOI : $10.4000 /$ lidil.70

ISSN : 1960-6052

\section{Éditeur}

UGA Éditions/Université Grenoble Alpes

\section{Édition imprimée}

Date de publication : 1 juin 2006

Pagination : 161-170

ISBN : 2-914176-14-7

ISSN : $1146-6480$

\section{Référence électronique}

Michel P. Schmitt, «École et dégoût littéraire », Lidil [En ligne], 33 | 2006, mis en ligne le 05 décembre 2007, consulté le 01 mai 2019. URL : http://journals.openedition.org/lidil/70 ; DOI : 10.4000/lidil.70

Ce document a été généré automatiquement le 1 mai 2019.

(c) Lidil 


\title{
École et dégoût littéraire
}

\author{
Michel P. Schmitt
}

1 À quoi sert un cours de littérature'? Qu'en reste-t-il dans le souvenir de ceux qui y ont assisté, et quelle place occupe-t-il dans le capital littéraire de chacun? Des entretiens ${ }^{2}$ personnalisés menés auprès de personnes ayant fréquenté le lycée, apportent quelques éléments de réponse en permettant d'observer un double phénomène. D'abord, la transformation mentale du temps investi dans l'activité sémiotique de la lecture, à partir de compétences linguistiques et axiologiques. Ensuite, l'énonciation des personnes interrogées, dans laquelle se confondent le texte lu, les impressions qu'il a laissées et les circonstances de sa lecture. Comme dans tout discours de croyance, l'énonciation fixe subjectivement les frontières du vrai et du faux, du bon et du mauvais, et s'élabore sans preuve. Nous appelons lectum le récit du souvenir de lecture, c'est-à-dire à la fois un «texte-lu » (désigné par un titre, une périphrase...) et la formulation même très fruste d'un affect concernant cet acte de lecture passé, l'implication singulière d'un être, à travers son discours, dans ses adhésions ou ses rejets. La trace des textes dans le récit des personnes interrogées est en effet le seul matériau valide et pertinent sur lequel puisse s'appuyer l'observateur. On mesure donc le lectum en objectivant des subjectivités singulières, en retenant dans le discours de l'enquêté ce qui est tenu pour lu, et que précèdent, accompagnent et suivent des jugements de valeur explicites ou implicites. C'est ce discours que nous voudrions mettre au jour, en nous en tenant ici à une population de lycéens qui, en septembre 2003 (trois mois après l'Epreuve Anticipée de Français du Baccalauréat), avaient dix-sept ans ${ }^{3}$.

2 Le discours qu'un individu est en mesure de tenir sur ses souvenirs littéraires ou culturels a socialement et historiquement évolué dans ses formes. C'est une évidence. Il suffirait de remonter trente ans en arrière pour apercevoir que les jeunes gens et jeunes filles d'aujourd'hui sont beaucoup plus nombreux à recevoir un enseignement littéraire obligatoire, qu'ils sont les agents déterminants d'une société de consommation culturelle dans laquelle les produits se sont multipliés et diversifiés de façon exponentielle, qu'ils lisent et commentent leurs lectures de livres de façon inédite. Le monde des croyances et des fétiches a changé, et on ne s'intéresse pas ici à la question de savoir si cette évolution est progrès ou déclin: on se contente d'enregistrer des variables, fragiles mais 
signifiantes, sans distinguer, dans le discours des personnes interrogées, le vrai du vraisemblable ou du bluff: c'est cette confusion même qui fonde le récit personnel du souvenir culturel qui toujours, lorsqu'il s'agit de donner une image de soi, se passe de référence à la preuve. Nous aimerions en même temps orienter notre recherche dans le sens d'une évaluation, quantitative et qualitative à la fois, des métamorphoses de l'objet littéraire quand on l'envisage du côté de ses réceptions. Tentons d'abord d'établir une cartographie des représentations des lectures, prescrites ou non. Une cartographie de ce que deviennent dans le récit des personnes interrogées, les corpus, modalités de lecture, enjeux, objectifs conjugués de l'institution scolaire et du marché du livre. Une cartographie du lectum, de cette anamorphose du référent qui le recrée dans les miroirs de la mémoire, et le traduit dans un idiolecte dont use la personne interrogée quand elle répond à l'enquêteur. Ces entretiens sont le premier moment d'un processus qui voudrait conduire vers une anthropologie de l'imaginaire susceptible de décrire la pratique sociale de la lecture comme une activité symbolique.

3 La dynamique du lectum s'opère dans trois directions contradictoires et complémentaires et conduit à distinguer trois ensembles. Un premier tiers des lecta s'agrège autour de 19 titres vedettes ( $4 \%$ du total des titres) ; un deuxième tiers rassemble quant à lui 432 titres ( $84 \%$ du total) ; un troisième et dernier tiers concerne 64 titres (12\% du total). Un même lecteur est potentiellement entrainé dans les trois mouvements à la fois, et c'est même le cas le plus fréquent. Le premier est centripète et donne de grandes chances de rejoindre un peu tout le monde sur 19 titres; le deuxième est centrifuge en se composant de titres fréquentés par un ou deux individus seulement ; le troisième est centripète à nouveau, mais à partir d'un consensus plus réduit. Chacun de ces trois tiers a sa structure propre. Le premier est très fortement induit par le marché culturel de masse, et les œuvres prescrites par l'institution scolaire y sont très minoritaires ${ }^{4}$; le deuxième est composé, comme le premier, d'ouvrages provenant du marché éditorial dans la partie qu'ignore la pratique scolaire; le troisième représente un corpus qui porte la trace manifeste des programmes et pratiques du collège et du lycée. En bref : le souvenir s'élabore à partir d'un très petit nombre de lecta qui proviennent des séries les plus célèbres de la bande dessinée enfantine et adolescente ; d'une poussière de titres dispersés en microscopiques espèces de capital à propriétaire unique; d'une poignée de classiques issus du collège et du lycée. Dans le récit qui en est fait au cours de l'entretien, les trois ensembles de lecta ne sont d'ailleurs pas rapportés de la même façon: le premier s'énonce sur le ton de l'évidence, comme allant de soi à partir du moment où on est assuré de ne pas encourir les moqueries de l'enquêteur en annonçant Tintin, Spirou ou Titeuf; les titres scolarisés du troisième ensemble s'intègrent pareillement dans des listes toutes faites, à nouveau sur le mode d'une évidence qui considère que l'enquêteur est nécessairement au courant de ce qui s'enseigne dans le secondaire ; mais les lecta du deuxième ensemble se livrent, pour leur part, comme des informations plus personnelles pour lesquelles l'enquêté(e) accepte d'ouvrir son jardin privatif. C'est pourquoi il est nécessaire, lorsqu'on fait l'inventaire de la bibliothèque d'un lecteur pris individuellement afin d'amorcer la topographie de ses goûts, d'apercevoir qu'à côté des classements par auteurs, titres, genres ou époques de production, on doit tenir le plus grand compte d'un dispositif général qui préexiste aux goûts de chacun et qui est valide pour le lectorat envisagé par l'enquête. Ici, une jeune fille de 17 ans en 2003 qui a lu Astérix s'agrège à la masse des gens de son âge; si elle a intégré dans son capital littéraire 1984, elle opère une distinction relative, en mentionnant un titre qu'affecte un nombre moins important de lecta mais qu'on relève 
encore fréquemment ; elle se distingue, en revanche, de tout le monde en citant Orgueil et préjugés. Le portrait d'un lecteur pris isolément est toujours composite, et tous les classements univoques sont illusoires.

4 La pratique différentielle des trois corpus définit aussi la structure temporelle et historique du souvenir, lui-même doublement affecté par l'oblitération du passé. Oubli qui affecte le passé de chacun et conduit la personne interrogée à citer d'abord des titres de son présent ou de son passé immédiat, en annulant des souvenirs plus anciens. Oubli ou méconnaissance de toute perspective historique d'autre part (à l'exception des didacta , fortement minoritaires) : 3 lecta sur 5 sont postérieurs à 1950. Les quatre années du xxie siècle couvrent la même surface que tout ce qui a été écrit de l'Antiquité à 1600 . Elles représentent 4 fois le xvie siècle, 2 fois tout le xviIIe. Ce qui appelle deux observations. D'abord, cela n'a pas toujours été le cas. Dans le corpus des souvenirs des personnes de plus de 60 ans en 2005, la place du passé littéraire (i.e. qui était déjà du passé quand elles avaient vingt ans) est beaucoup plus forte. Ensuite, ce passé qui s'identifie avec les corpus institués, en revêt également les énonciations : tandis que le lectum non prescrit se raconte comme un rapport personnalisé avec un texte, le didactum confond l'œuvre, sa fonction diplômante (son rôle d'évaluation dans le cadre d'un programme) et la personnalité de l'enseignant(e) en charge du cours. Cette confusion épistémologique façonne et renforce les matrices historico-génériques du souvenir littéraire: les lecta dramatiques sont des didacta du Xvile siècle (une petite dizaine de pièces de Molière et de Racine); les lecta poétiques sont des didacta du xixe siècle (Baudelaire, Verlaine et Rimbaud). Les lecta qui relèvent de la littérature d'idées sont des didacta du XviIIe. Quant aux lecta de prose narrative, et même si les corpus ont évolué, leur matrice formelle demeure celle du xixe siècle (Hugo, Zola, Maupassant).

5 Le peu d'appétence personnelle pour le passé s'accompagne dans les lecta d'un désintérêt pour un corpus d'œuvres de langue française qui mêlent un référent national (personnages, décors, histoire et société...) avec une langue dans son génie propre. Le tropisme pour l'étranger anglo-saxon dans le lectum non institué est en revanche fortement marqué. Non qu'il s'agisse de textes dans leur version originale (les lecta se font sur des traductions), mais parce que les référents renvoient faiblement ou pas du tout à des realia proprement nationaux, et que le français y apparait comme un code linguistique purement utilitaire. Le souvenir littéraire n'est donc pas celui de l'actualisation linguistique et stylistique d'un référent: il porte sur des codes linguistiquement français, syntaxiquement médiatiques, symboliquement attachés au compte rendu d'un présent réel ou fantasmé. On en prendrait pour preuve le cas extrême des lecta de textes versifiés. Présents, même de façon modeste, ils renvoient à des états psychologiques, une philosophie de l'existence, un ethos, sans être jamais accompagnés de vers qu'on connaitrait " par cœur » par exemple. Le phénomène ne peut être mis sur le compte de la mémoire collective contemporaine qui se soucierait peu du "par cœur » : il suffit d'observer le nombre considérable de chansons dont les jeunes filles connaissent parfaitement les paroles, pour comprendre que dans le souvenir de la poésie instituée, quand il est heureux, les fonctions sémantique et symbolique sont privilégiées au détriment des fonctions phatique, linguistique et poétique.

Ces souvenirs mettent en lumière la pratique des genres littéraires. Plus des trois-quarts appartiennent à la prose narrative, ce qui est sans surprise, mais doit se détailler. Commençons a contrario par le quart qui formellement n'appartient pas à la prose narrative. Théâtre, poésie, littérature d'idées : tous ces lecta ont été induits par l'école. 
Les titres qui relèvent de ces macrogenres mais que ne prend pas en charge l'école n'occupent aucune place dans l'imaginaire de ces jeunes filles et jeunes gens. C'est aussi pour eux que les repulsa' sont les plus nombreux et les plus fortement marqués par l'ennui indifférent ou le commentaire désobligeant. Tout se passe comme si le filtre de la mémoire ne retenait que la prose narrative, pour se détourner du reste, ou n'en garder que ce qui dans la pratique obligatoire a été suffisamment désagréable pour ne pas avoir été oublié. Distinguons alors dans le macrogenre dominant de la prose narrative, des sous-ensembles propres à mieux dégager les reliefs de notre cartographie. La bande dessinée pour enfants et d'aventures vient en tête dans le récit (heureux) de lecture. La prose narrative française suit. À elles deux, elles couvrent la moitié de l'ensemble. La deuxième moitié est constituée par la prose narrative anglo-saxonne immédiatement contemporaine, le thriller, le roman policier à « énigme » et dans une mesure infime le roman d'aventures. Dans ces ensembles, la logique de la prescription scolaire interfère avec celle qui a cours hors de l'institution, pour dessiner un palmarès hybride de lecta qui donne des équivalences classiques inattendues. Molière par exemple partage la première place avec Goscinny. Voltaire est $7^{\mathrm{e}}$, comme Roba. Hugo et Higgins Clark se partagent la $9^{\mathrm{e}}$ place. Les $11^{\mathrm{e}}$ sont 5 ex-aequo : La Fontaine, Zola, Van Hamme, Zep et Werber. Maupassant est $16^{\mathrm{e}}$, Cauvin aussi. Les $18^{\mathrm{e}}$ Baudelaire et Racine sont rejoints par Tolkien et Nothomb. Et les $21^{\mathrm{e}}$ Montesquieu, Rousseau et Sartre accueillent leurs homologues Tomme et Janry, Rosinski et « les mangas ».

7 La morphologie générale du souvenir littéraire laisse apercevoir d'autres clivages, plus classiques. Passons rapidement sur le premier de ces clivages, fondamental mais connu de tous : celui de l'origine socio-familiale des individus, que souligne, structure et renforce l'orientation scolaire dans les sections de l'enseignement secondaire. Ce n'est pas parce que tel individu est passé par telle section que son capital littéraire prend une forme particulière; ce sont au contraire ses compétences linguistiques et ses références culturelles acquises antérieurement et hors de l'école qui le préparent, voire le destinent, à se retrouver dans des sections où l'enseignement littéraire ne pèse pas le même poids et n'a pas les mêmes finalités. Ne nous étonnons pas trop si on a davantage de chances de trouver la trace du De amicitia chez une jeune fille ex-élève de section L, que chez les exélèves masculins des sections technologiques qui se reconnaissent dans la série des XIII et dans les mangas. Moins souvent analysé : le clivage sexuel. Les jeunes filles mémorisent un capital plus important, de façon plus détaillée et selon des cheminements plus structurés. Il est vrai que filles et garçons contribuent à parts égales à la constitution du peloton de tête des 19 œuvres les plus référentielles : bande dessinée distrayante pour enfants, thrillers, trois classiques scolaires. Tintin est consensuel, Titeuf ou Dom Juan, Lucky Luke ou Candide, Le Malade imaginaire aussi : tous sont des lecta bisexués. Mais il existe des chasses sexuellement gardées, que l'on observe en classant les titres vedettes de l'un et l'autre sexe. Tandis que Boule et Bill, Spirou, Gaston Lagaffe, Tolkien, Thorgal, Stephen King (et Astérix dans une large mesure) délimitent le territoire des jeunes gens, les jeunes filles s'agrègent plutôt entre elles autour d'Agatha Christie ou d'Higgins Clark, des Confessions de Rousseau (pour en dire beaucoup de mal), d'Harry Potter, du Petit Spirou ou de l'Avare. À cet égard, les clichés en termes d'ethos textuel se confirment: le territoire masculin s'organise autour d'auteurs exclusivement masculins, de personnages éponymes masculins, dans le registre drôlatique et de l'action guerrière (Gaston Lagaffe, Spirou, Le Seigneur des anneaux, Thorgal) et il n'empiète jamais sur le didactum. Sur le territoire féminin en revanche se réunissent 3 auteures sur les 7 écrivains considérés (Higgins 
Clark, Christie, Rowling, dans un peloton que complètent Molière, Tomme et Janry, Rousseau et Voltaire) ; les héros sont plus variés, et à défaut d'être toujours féminins, ils sont volontiers des enfants (Le Petit Spirou, Titeuf, Harry Potter) ; 4 ont aussi un statut de didacta. Le souvenir littéraire féminin par ailleurs construit un ensemble de lecta 1,5 fois plus important, établi d'une façon plus originale. Les lecta étrangers représentent $40 \%$ des lecta des deux sexes confondus (pourcentage très important au regard du didactum étranger en France, voisin de zéro), mais on trouve une double supériorité féminine, autant dans l'exploration des lecta étrangers qu'on trouve avec une seule occurrence $(1,5$ fois plus forte), que celle des lecta français du même type (2 fois plus forte).

8 En même temps que les déterminations socio-scolaires des individus, interviennent les conditions de réalisation d'un sens à travers la lecture, liées à l'organisation de l'emploi du temps de chacun. Après le nécessaire détour par la cartographie, il faut alors appréhender le lectum comme une mythographie de chacun par lui-même. Une autofiction en quelque sorte, qui permet à l'individu de se (re)constituer une histoire personnelle. Car ne nous trompons pas. Ce n'est pas parce que le livre est devenu un objet passablement obsolète, que le multimédia a engendré une civilisation chargée de sémiologies nouvelles et que l'illettrisme progresse à pas de géant, qu'il faudrait penser que le lectum ne sait plus rien dire de la société actuelle. Les jeunes gens et jeunes filles de 17 ans en 2005, qui viennent d'en finir avec la littérature instituée (mis à part ceux qui en feront leur spécialité), sont les chefs et sous-chefs de demain : leur lectum se présente en fait comme un lieu privilégié pour observer et comprendre le politique aujourd'hui. Les usages du littéraire, parmi lesquels il faut au premier rang compter la fonctionnalité scolaire d'un corpus clos, renseignent sur les modes de l'aliénation de masse dans la postmodernité. Celle-ci ne passe plus par l'imposition univoque de dogmes ou de fétiches, mais par l'atomisation de produits interchangeables, classés par l'institution scolaire ou le marché culturel. Les pratiques de la lecture sont donc prises - sauf pour un groupe microscopique de clercs et de curieux - entre les choix téléguidés par le faux hasard des rencontres organisées de la marchandise, et le respect formel dû aux titres et écrivains institués. Entre des produits présentés comme l'objet (peu obscur) du désir facile, et l'ennui institutionnellement rémunérateur.

Les notions fondamentales de l'analyse textuelle s'en trouvent violemment bouleversées. « Auteur ", « œuvre », " genre », et même histoire des esthétiques, autant de termes qui acquièrent de nouvelles définitions. La répartition de la propriété littéraire symbolique doit se faire d'une toute autre façon, et il est même probable que le "champ littéraire " doive s'envisager dans une perspective différente. Ce qui amène à s'interroger sur les «classiques» de la pratique et non ceux de la révérence scolaire. Parmi les 300 titres isolés (qui n'ont droit qu'à un seul lectum), on ne trouve que 70 didacta potentiels. La quête solitaire, celle qui n'est pas portée par un mouvement collectif de mode ou par une prescription forte, se porte pour plus des 3/4 sur autre chose que la littérature instituée. En revanche, les titres autour desquels se dessine un consensus moyen font apparaitre 3/4 de didacta potentiels. Enfin, les 19 œuvres pour lesquelles se crée un fort consensus n'appartiennent qu'à hauteur de 1 pour 5 au fonds de la littérature scolaire. En d'autres termes, la découverte solitaire ou l'agrégation à un consensus fort se détournent l'une et l'autre $\mathrm{du}$ fonds de la lycératures. Les efforts considérables de l'institution pour transmettre le didactum se soldent par un échec mesurable à l'aune de l'oubli et de l'indifférence qui le recouvrent. L'école induit une appropriation littéraire consensuelle c'est une de ses missions explicites - mais globalement dépréciée ou, plus souvent encore, 
largement reçue dans l'indifférence. Etrangère à la fois aux explorations singulières et aux mouvements de masse du goût, elle conforte une institution mais échoue à construire une culture littéraire.

On peut alors esquisser une sociopoétique qui serait fondée sur la catégorie du lectum comme matrice de sens, en observant que les lecta fondent des catégories spécifiques à partir de critères explicitement émotionnels et affectifs, et fort peu historiques, éthiques ou idéologiques. Les trois catégories dominantes qui fonctionnent comme des matrices de sens a priori sont le « divertissant ", l'« intéressant » et le "scolaire ». Dans les trois cas, l'effet de lecture s'est réalisé à partir de fonctionnalités qui nourrissent le discours social et s'en nourrissent. S'en trouvent alors disqualifiés la pensée discursive (brutalement confondue avec l'exercice scolaire obligatoire), le référent historique, la notion d'œuvre comme clôture, et l'attention portée à la langue et au style. Ces divers aspects sont pourtant ceux qui fondent, à bon droit, bien que de façon variable et parfois ambigüe, l'enseignement littéraire. La lycérature atteint donc les objectifs exactement à l'inverse de ceux qu'elle prétend se donner et prend pour finir, dans l'ensemble complexe du lectum, la part de l'illisible. Elle conduit jeunes filles et jeunes gens à identifier le littéraire au métadiscours académique, sans autre enjeu qu'une épreuve d'examen sur des objets présélectionnés par une institution autarcique. Elle apprend en fait à ne pas lire. La « responsabilité des formes » dont parle Barthes, serait donc à réévaluer à travers la double énonciation du didactum, qui ne rend pas compte des textes littéraires de façon critique et esthétique, mais renvoie aux conditions et situations objectives de leur enseignement.

\section{NOTES}

1. On entend ici par « cours de littérature » celui qui est dispensé à tous les lycéens, et non celui que suivent les sections spécialisées, les classes préparatoires littéraires, etc. Par ailleurs, notre recherche ne prend tout son sens que dans la perspective des recherches menées autour de la littérature telle qu'elle s'enseigne au lycée. On ne peut ignorer, notamment, les ouvrages fondamentaux d'Anne-Marie Chartier et Jean Hébrard ( Discours sur la lecture : 1880-1980, Fayard-BPI, 2000), Bernard Veck (La Culture littéraire au lycée : des humanités aux méthodes? INRP 1995), Martine Jey (La Littérature au lycée : invention d'une discipline (1880-1925), Centre d'étude et de linguistique des textes et des discours, Coll. Recherches textuelles, 1998), ou Jean-François Massol (De l'institution scolaire de la littérature française (1870-1925), Ellug, 2004).

2. Entre 2001 et 2005 ont été réalisées dans le cadre du séminaire de recherche " Littérature, lectures, lecteurs » (Faculté des Lettres, Université Lumière-Lyon 2) 5 enquêtes, organisées par Michel P. Schmitt et prises en charge par les étudiants. Ces enquêtes s'appuient sur des entretiens personnalisés et approfondis (une demi-heure à une heure). Plusieurs centaines d'entretiens ont ainsi été rassemblés, selon la méthode des quotas, sur des échantillons construits à partir de la nomenclature des catégories socio-professionnelles telle que l'analysent Alain Desrosières et Laurent Thévenot dans 
Les catégories socio-professionnelles (Editions La Découverte, 2000). L'ORTEIL (Observatoire des réceptions, transmissions, enseignements et institutionnalisations de la littérature) a poursuivi le travail de collecte et d'interprétation mené par les étudiants.

3. Soixante entretiens ont été réalisés en 2003 par 20 étudiants du séminaire auprès de garçons et de filles de 17 ans, soit l'âge où, quel que soit le passé scolaire de chacun, on en a fini avec l'enseignement obligatoire du français littéraire. Les personnes interrogées étaient issues de toutes les sections du lycée. Nous avons respecté la proportion de chacune dans l'ensemble des classes de Première (la Direction Programmation et Développement de l'Education Nationale fournit les chiffres nécessaires). Ont été contactés : 1 garçon et 7 filles de L; 11 garçons et 9 filles de $\mathrm{S} ; 4$ garçons et 6 filles de ES ; 4 garçons et 6 filles de STT ; 4 garçons et 1 fille de STI ; 3 filles de SMS et STL ; 2 garçons et 2 filles de 1re d'adaptation. Chaque enquêteur s'est appuyé sur un guide d'entretien délibérément souple. Il s'est centré sur les souvenirs littéraires des personnes interrogées, en réunissant des titres et des auteurs qu'elles disaient avoir fréquentés. Il a dans toute la mesure du possible suscité un commentaire appréciatif ou dépréciatif, des renseignements sur l'historique des lectures, etc. Les étudiants sont ensuite passés à la phase de caractérisation littéraire des 515 titres ainsi collectés, répartis sur 1298 lecta. 4. Il s'agit de Dom Juan, Candide, Le Malade imaginaire, des Confessions et de L'Avare. 5. Les étudiants-enquêteurs se présentaient comme tels auprès des personnes qu'ils interrogeaient.

6. Le didactum désigne un texte dès lors qu'il a été prescrit par l'institution scolaire. La liste des didacta s'établit à partir des programmes officiels et des déclarations des enquêtés eux-mêmes.

7. Le repulsum désigne un texte sur lequel les personnes interrogées portent un jugement négatif explicite.

8. Ce mot-valise sert à désigner la littérature telle qu'elle est enseignée au lycée.

\section{RÉSUMÉS}

À l'issue de la classe de première, quels souvenirs reste-t-il de l'enseignement littéraire amorcé dès le collège (voire l'école primaire) et renforcé au lycée ? Comment ces souvenirs s'intègrent-ils dans la partie littéraire du capital culturel de chacun de ces jeunes filles et de ces jeunes gens aujourd'hui ? La «bibliothèque lue » se répartit en trois ensembles différents, qui témoignent de formes très variées d'adhésion aux œuvres, et dans lesquels les titres scolarisés sont dans tous les cas très minoritaires. Les œuvres du passé (antérieures à 1950) couvrent à peine $2 / 5^{\mathrm{e}} \mathrm{de}$ l'ensemble, et ce sont sur elles, comme sur les œuvres françaises en général, que portent les jugements les plus défavorables. Les bibliothèques lues sont très différentes selon les sections considérées et mettent en évidence des pratiques et des goûts très différents qui recoupent l'origine socio-familiale et le sexe des personnes interrogées. L'étude de la réception littéraire fondée sur la notion de lectum conduit à reconsidérer les notions d' "auteur ", de "genre » et d'« œuvre», et aussi à s'interroger de façon radicale sur les modalités de l'enseignement littéraire au lycée. 
AUTEUR

MICHEL P. SCHMITT

Université Lumière-Lyon 2. 